\title{
Паранепротиворечивые категории для паранепротиворечивой логики
}

\author{
В. Л. ВАСЮКОВ
}

\begin{abstract}
It is well known that the concept of da Costa algebra [4] reflects most of the logical properties of paraconsistent propositional calculi $C_{n}(1 \leq n \leq \omega)$ introduced by N.C.A. da Costa. In [8] the construction of topos of functors from a small category to the category of sets was proposed which allows to yield the categorical semantics for da Costa's paraconsistent logic. Another categorical semantics for $C_{n}$ would be obtained by introducing the concept of potos - the categorical counterpart of da Costa algebra (the name "potos" is borrowed from W.Carnielli's story of the idea of such kind of categories). In the paper the potos completeness of da Costa logics (i.e. in respect to the potoses) is proved.

Ключевые слова: алгебра да Косты, паранепротиворечивые категории, потосы, общезначимость, основания теории категорий, комплементарно замкнутые категории
\end{abstract}

\section{1 Введение}

Как известно, концепция алгебры да Косты [4] отражает большинство логических свойств паранепротиворечивого пропозиционального исчисления $C_{n}, 1 \leq n \leq \omega$, введенного впервые H.K.А. да Костой. В работе [8] автор предложил конструкцию топоса функторов из малой категории в категорию множеств, которая позволяет получить категорную семантику для паранепротиворечивой логики да Косты. Другая категорная семантика для $C_{n}$ могла бы быть получена путем рассмотрения концепции nотоса или топоса да Косты - категорного эквивалента алгебры да Косты.

Потос представляет собой паранепротиворечивый универсум, в котором можно было бы развивать паранепротиворечивую математику, подобно тому как это делается в топосах для интуиционистской математики. Но если в [8] все случаи паранепротиворечивости возникают лишь как частные конструкции в интуиционистском универсуме, как некоторые локальные артефакты, 
то в потосе эта паранепротиворечивость абсолютно органична и, более того, она лежит в основе всех конструкций, она глобальна и фундаментальна. Здесь уже классическая математика возникает как артефакт в паранепротиворечивом универсуме, как некоторое локальное отклонение от паранепротиворечивых закономерностей. Так, например, при интерпретации систем $C_{n}$ можно использовать неистинностно-функциональную оценку, в то время как истинностно-функциональная оценка становится характерной только для случая булевых топосов, которые являются теперь всего лишь частным случаем потосов.

В работе [1] автором уже была предложена конструкция потоса как декартово замкнутой категории (с начальным объектом 0 и терминальным объектом 1), содержащей выделенный объект $\Omega$, который имплицитно является алгеброй да Косты, т.е. имеются стрелки true $: 1 \rightarrow \Omega$, false $: 1 \rightarrow \Omega, \overline{\rceil}: \Omega \rightarrow \Omega, \cap: \Omega \times \Omega \rightarrow$ $\Omega, \cup: \Omega \times \Omega \rightarrow \Omega, \supset: \Omega \times \Omega \rightarrow \Omega$, выполняющие условия алгебры да Косты из работы $[4$, p. 81]. Однако подобное определение потоса страдает тем недостатком, что истинностная стрелка отрицания, в отличие от других стрелок, вводится «локально» и не связана с какими-либо категорными конструкциями.

В данной работе преодолевается этот недостаток путем введения так называемой комплементарной замкнутости декартово замкнутой категории. Это позволяет строить истинностную стрелку отрицания «глобально», по образцу введения остальных истинностных стрелок.

Следствием новой конструкции топоса является рассмотрение новой категории паранепротиворечивых множеств PSet, когда в качестве объектов используются множества системы $Z F_{1}$ паранепротиворечивой теории множеств, которая соотносится с теорией множеств Цермело-Френкеля $Z F_{0}$ так же, как паранепротиворечивая первопорядковая логика с равенством соотносится с классической первопорядковой логикой с равенством. При этом категория PSet оказывается не топосом, а потосом множеств.

Основание подобного рассмотрения можно найти у Ж. Бенабу, который в [3] предлагал в качестве минимальных теоретикомножественных оснований теории категорий рассматривать теорию множеств как любую теорию, использующую язык $Z F_{0}$, и 
использующую всего лишь аксиому экстенсиональности и аксиому выделения. Для любой модели подобной теории ее элементы будут представлять собой множества, в то время как «метамножества» универсума дискурса, откуда выбирается модель, будут представлять собой классы. Следуя Бенабу, мы можем определить требуемую нам теорию множеств как любую теорию, использующую язык паранепротиворечивой теории множеств $Z F_{1}$, и выбрать любую модель подобной теории в универсуме дискурса (фактически расширяя наш универсум до универсума всех неклассических «метамножеств»). Тогда так называемое отображение Йонеды будет сопоставлять каждому множеству некоторый его класс-представитель относительно нашей «паранепротиворечивой» модели, а наши множества будут в точности теми множествами, которые нужны нам для рассмотрения категории PSet.

Во втором параграфе приводятся минимальные сведения из теории алгебр да Косты, которые требуются для дальнейшего изложения, поскольку последние существенным образом используются и в значительной степени детерминируют саму конструкцию потоса.

В третьем параграфе вводится понятие потоса, анализируются его свойства и требуемые теоретико-множественные основания и рассматривается потос PSet. Вводятся также конструкции истинностных стрелок в произвольном потосе.

В четвертом параграфе дается алгебраическая интерпретация системы логики да Косты $C_{1}$ в алгебрах да Косты и теоретикопотосная интерпретация этой системы, существенно основанная на этих алгебрах. Доказывается полнота этой логической системы относительно данных интерпретаций. Наряду с этим рассматривается неистинностно-функциональная оценка для системы $C_{1}$ и полнота данной системы доказывается также с использованием этой оценки.

\section{2 Алгебры да Косты}

В 1984 г. В.А. Карниелли и Л.П. Алькантара [4] сформулировали понятие алгебры да Косты, отражающей большинство логических свойств паранепротиворечивой логики $C_{n}$. Было показано, что алгебра да Косты изоморфна паранепротиворечи- 
вой алгебре множеств, что можно было бы рассматривать как аналог стоуновского представления для булевой алгебры. Однако подобная аналогия правомочна только с неклассической точки зрения: некоторые из операций в паранепротиворечивой алгебре множеств не сформулированы в обычных теоретикомножественных терминах.

Поскольку наши теоретико-категорные конструкции в дальнейшем будут существенно основаны на алгебрах да Косты, то для дальнейших построений приведем требуемые нам полные определения.

ОПРЕДЕЛЕНИЕ 1. [4, p. 81] Под алгеброй да Косты будем понимать структуру $A=\left\langle S, 0,1, \leq, \wedge, \vee, \supset,^{\prime}\right\rangle$ такую, что для каждого $a, b, c$ из $S$ выполняются следующие условия:

1. $\leq$ есть предпорядок;

2. $a \wedge b \leq a, a \wedge b \leq b$;

3. если $c \leq a$ и $c \leq b$, то $c \leq a \wedge b$;

4. $a \wedge a=a, a \vee a=a$;

5. $a \wedge(b \vee c)=(a \wedge b) \vee(a \wedge c)$;

6. $a \leq a \vee b, b \leq a \vee b$;

7. если $a \leq c$ и $b \leq c$, то $a \vee b \leq c$;

8. $a \wedge(a \supset b) \leq b$;

9. если $a \wedge c \leq b$, то $c \leq(a \supset b)$;

10. $0 \leq a, a \leq 1$;

11. $x^{o} \leq\left(x^{\prime}\right)^{o}$, где $x^{o}=\left(x \wedge x^{\prime}\right)^{\prime}$;

12. $x \vee x^{\prime} \equiv 1$, где $a \equiv b$ тогда и только тогда, когда $a \leq b$ и $b \leq a$;

13. $x^{\prime \prime} \leq x$, где $x^{\prime \prime}$ означает $\left(x^{\prime}\right)^{\prime}$;

14. $a^{o} \leq(b \supset a) \supset\left(\left(b \supset a^{\prime}\right) \supset b^{\prime}\right)$;

15. $x^{o} \wedge\left(x^{o}\right)^{\prime} \equiv 0$.

Если существует $x \in S$, такой, что неверно, что $x \wedge x^{\prime} \equiv 0$, то про алгебру $A$ говорят, что она является собственной алгеброй да Koсmu.

ПРЕДЛОЖЕНИЕ 1. [1, р. 82] Если $A=<S, 0,1, \leq, \wedge, \vee, \supset,{ }^{\prime}>$ есть алгебра да Косты, то справедливы следующие утверждения:

$(C 1) y \leq x$ тогда и только тогда, когда $x \wedge y \equiv y$;

$(C 2) x \wedge 0 \equiv 0, x \vee 1 \equiv 1$ 
(C3) $x \vee 0 \equiv x, x \wedge 1 \equiv x$;

(C4) $x \vee y \equiv y \vee x, x \wedge y \equiv y \wedge x$

(C5) если $x=y$, то $x \equiv y$;

(C6) если $a \equiv b u x \equiv y$, mo $x \wedge a \equiv y \wedge b$;

(C7) если $a \equiv b u x \equiv y$, mo $x \vee a \equiv y \vee b$;

(C8) $y \leq x$ тогда и только тогда, когда $y \vee x \equiv x$;

(C9) если $x \vee y \equiv 0$, то $x \equiv 0$ u $y \equiv 0$;

$(C 10)$ eсли $x \leq y^{o}$ u $x \leq\left(y^{o}\right)^{\prime}$, mo $x \equiv 0$;

$(C 11) p \vee\left(p^{\prime} \wedge p^{o}\right) \equiv 1, p^{\prime} \vee\left(p^{\prime} \wedge p^{o}\right) \equiv 1$

(C12) $x \wedge y \equiv 0$ тогда и только тогда, когда $x \leq y^{\prime} \wedge y^{o}$;

$(C 13) x \wedge\left(y \wedge y^{o}\right) \equiv 0$ тогда и только тогда, когда $x \leq y^{\prime}$;

(C14) $x \wedge x^{\prime} \wedge x^{o} \equiv 0$

$(C 15)$ если $x \wedge\left(x^{\prime}\right)^{o} \equiv 0$, mo $x \equiv x^{\prime \prime}$;

(C16) если $x^{\prime} \wedge\left(x^{\prime}\right)^{o} \equiv 0$, mo $x \equiv x^{\prime \prime}$;

(C17) если $x \wedge y \equiv 0$, mо $x \leq y^{\prime}$

(C18) если $y \equiv y^{o}$, то $x \wedge y \equiv 0$ тогда и только тогда, когда $x \leq y^{\prime}$

(C19) $x \wedge y^{\prime} \wedge y^{o} \equiv 0$ тогда и только тогда, когда $x \leq y$.

Доказательство. Очевидно.

Q.E.D.

ПРЕДЛОЖЕНИЕ 2. [1, р. 83] Каждал алгебра да Косты имеет по крайней мере три элемента.

ОПРЕДЕЛЕНИЕ 2. [1, р. 83] Паранепротиворечивая алгебра множеств представляет собой структуру $A=\left\langle S, \varnothing, I, \leq, \cap, \cup, \Rightarrow,{ }^{\prime}\right\rangle$, где

1. $\cap u \cup$ являются операциями пересечения и объединения;

2. $\leq$ есть предпорядок;

3. $S \subseteq \wp(I)$;

4. $S$ замкнуто относительно бинарных операций $\cap, \cup$ и унарной операции ';

5. $a \cap b \leq a, a \cap b \leq b$;

6. если $c \leq a$ и $c \leq b$, то $c \leq a \cap b$;

7. $a \leq a \cup b, b \leq a \cup b$;

8. $a \cap(a \Rightarrow b) \leq b$;

9. если $a \cap c \leq b$, то $c \leq(a \Rightarrow b)$;

10. $\varnothing \leq a, a \leq I$; 
11. $x \cup x^{\prime} \Leftrightarrow I$, где $a \Leftrightarrow b$ тогда и только тогда, когда $a \leq b$ и $b \leq a$;

12. $x^{\prime \prime} \leq x$;

13. $x^{o} \leq(y \Rightarrow x) \Rightarrow\left(\left(y \Rightarrow x^{\prime}\right) \Rightarrow y^{\prime}\right)$, где $x^{o}=\left(x \cap x^{\prime}\right)^{\prime}$;

14. $x^{o} \cap\left(x^{o}\right)^{\prime} \Leftrightarrow \varnothing$.

15. $x^{o} \leq\left(x^{\prime}\right)^{o}$.

Пусть $S_{0}=\left\{x: x \in S\right.$ и $\left.x \cap x^{\prime} \nRightarrow \varnothing\right\}$. Если $S_{0} \neq \varnothing$, то мы имеем собственную паранепротиворечивую алгебру да Косты. Каждая паранепротиворечивая алгебра множеств является собственной алгеброй да Косты, в то время как каждая булева алгебра множеств является несобственной алгеброй множеств.

Для данного отношения эквивалентности про две алгебры да Косты $A$ и $B$ говорится, что они будут - изоморфными, если имеется функция $f$ из $A$ на $B$, сохраняющая операции и являющаяся -инбективной, т. е., когда если $x \nsim y$, то $f(x) \neq f(y)$.

TEOPEMA 1. [4, p. 84] Каждая собственная алгебра да Кость 三-изоморфна собственной паранепротиворечивой алгебре множеств.

В [8, p. 273] доказана следующая теорема:

TEOPEMA 2. Множество максимальных фильтров алгебры да Кость 三-изоморбно алгебре да Косты.

\section{3 Потосы}

Потос фактически представляет собой топос, наделенный некоторой дополнительной структурой. По сути дела мы могли бы говорить не о потосах, а о «паранепротиворечивых топосах» или «топосах да Косты». Термин «потос» был навеян рассказом В. Карниелли об идее подобной разновидности категорий, высказанной одним из бразильских математиков, который именно так называл эти гипотетические категории.

ОПРЕДЕЛЕНИЕ 3. Потос $C$ является декартово замкнутой категорией, которая комплементарно замкнута и имеет классификатор подобъектов.

Комплементарная замкнутость $C$ означает, что

(i) для любого объекта $a$ в $C$ имеется объект $a^{\prime}$, такой, что в $C$ существуют стрелки $a^{\prime \prime} \rightarrow a$ и $a^{o} \rightarrow\left(a^{\prime}\right)^{o}$, где $a^{o}=\left\langle a, a^{\prime}\right\rangle^{\prime}$, 
(ii) $1 \cong\left[a, a^{\prime}\right], 0 \cong\left\langle a^{o}, a^{o \prime}\right\rangle$,

(iii) для любых двух объектов в $C$ имеется стрелка $a^{o} \rightarrow(b \Rightarrow$ $a) \Rightarrow\left(\left(b \Rightarrow a^{\prime}\right) \Rightarrow b^{\prime}\right)$, где $b \Rightarrow a$ есть экспоненциал.

ПРЕДЛОЖЕНИЕ 3. В потосе С совокупность $S$ И $(d)$ всех мономорфных С-стрелок с концом d является алгеброй да Косты.

Доказательство. Поскольку любой потос $C$ является декартово замкнутой категорией, то для любого объекта $d$ в $C$ совокупность $S u b(d)$ всех мономорфных $C$-стрелок с концом $d$ образует предупорядоченную дистрибутивную решетку. Это означает, что условия 1-7 и 10 из определения алгебры да Косты выполняются в $S u b(d)$. Условия 8-9 являются следствиями диаграммы экспоненцирования. Условия (i)-(iii) определения потоса гарантируют нам выполнение условий 11-15.

Q.E.D.

Нетрудно видеть, что в потосе мы имеем $\operatorname{Sub}(d) \cong \operatorname{Hom}(d, \Omega)$ и таким образом $\operatorname{Hom}(d, \Omega)$ будет представлять собой алгебру да Косты. Но в этом случае возникает проблема категории множеств Set. Дело в том, что в Set мы имеем $\operatorname{Sub}(D) \cong \wp(D)$, где $\wp(D)=\{x: x$ является подмножеством множества $D\}$. Поскольку $\wp(D)$ есть булева алгебра подмножеств, а не паранепротиворечивая алгебра множеств, как можно было бы ожидать по теореме 1 , то приходим к заключению, что Set не может быть потосом. Но согласно определению паранепротиворечивой алгебры множеств существуют множества, образующие подобную алгебру. Таким образом, либо подобные множества порождают подкатегоррию PSet категории $S e t$, либо $S e t$ в каком-то смысле является подкатегорией PSet.

Известно (см. [7]), что существует система $Z F_{1}$ паранепротиворечивой теории множеств, которая соотносится с теорией множеств Цермело-Френкеля $Z F_{0}$ так же, как паранепротиворечивая первопорядковая логика с равенством $C_{1}^{=}$соотносится с классической первопорядковой логикой с равенством $C_{0}^{=}$. В сущности « $Z F_{1}$ должна быть 'частично' включенной в $Z F_{0}$, хотя последняя также содержится в некотором смысле в первой» $[7$, p. 170]. Базисные теоретико-множественные понятия $Z F_{1}$ аналогичны соответствующим понятиям $Z F_{0}$, хотя понятия, использующие отрицание, порождают два вида понятий: одни со сла- 
бым отрицанием $(\neg)$, а другие с сильным отрицанием $\left(\neg^{*}\right.$, полагая $\left.\neg^{*} A=d_{\text {ef }} \neg A \wedge A^{o}\right)$. Как следствие, мы имеем, например, два пустых множества: $\varnothing=\{x: x \neq x\}$ и $\varnothing^{*}=\left\{x: \neg^{*}(x=x)\right\}$.

Совокупность всех множеств плюс $\varnothing, V, \cap, \cup{ }^{C}$ (где $V=\{x$ : $x=x\}$ и $\left.x^{C}=\{y: y \notin x\}\right)$ образует в $Z F_{0}$ полную булеву алгебру. Для $Z F_{1}$ мы получаем следующий результат:

ПРЕДЛОЖЕНИЕ 4. В $Z F_{1}$ совокупность всех множеств плюс $\varnothing, V, \cap, \cup{ }^{C}$ образует паранепротиворечивую алгебру множеств.

Доказательство. Непосредственной проверкой (полагая $x \Rightarrow$ $y=\{z: z \in x \rightarrow z \in y\})$.

Q.E.D.

Каждая схема аксиом $Z F_{0}$ порождает соответствующие две схемы аксиом $Z F_{1}$, одну с сильным отрицанием, а другую со слабым. Таким образом, мы можем сказать, что $Z F_{1}$ включает в себя $Z F_{0}$ и, следовательно, Set действительно в некотором смысле является подкатегорией PSet. Но что означает существование категории множеств, отличной от Set?

Ж. Бенабу в [3, p. 18], пытаясь выявить минимальные теоретико-множественные основания теории категорий, определяет теорию множеств как любую теорию $T$, использующую язык $Z F_{0}$, и использующую, по меньшей мере, аксиому экстенсиональности $E$ и аксиому выделения $C S$. Пусть для любой модели $M$ подобной теории элементы $M$ будут называться множествами и обозначаться как $S, T, \ldots$, а формальные отношения принадлежности и равенства множеств будут обозначаться как $S \in \in^{*} T$ и $S \stackrel{\circ}{=}$. Тогда «метамножества» универсума дискурса $\mathbf{U}$, откуда выбирается модель $M$, будут называться классами и обозначаться как $\mathbf{S}, \mathbf{C}, \ldots$, в то время как отношения прнадлежности и равенства в $\mathbf{U}$ будут обозначаться обычными символами $\in$ и $=$. Подкласс $\mathbf{S}$ из $M$ будет представимым, если имеется множество $S$, называемое представителем $\mathbf{S}$, такое, что для всех $T \in M$ мы имеем $T \in \mathbf{S}$ тогда и только тогда, когда $T \in \in^{*} S$. Отображение Йонеды сопоставляет каждому множеству $S$ класс-представитель $\hat{S}=\left\{T \in M: T \in^{*} S\right\}$.

Аксиома экстенсиональности будет, например, в этом случае читаться следующим образом:

(Е) Для всех множеств $S$ и $T, \hat{S}=\hat{T}$ тогда и только тогда, когда $S \stackrel{\circ}{=}$. 
Следуя Бенабу, мы можем определить теорию множеств как любую теорию $T$, использующую язык $Z F_{1}$, и выбрать любую модель $M$ подобной теории в универсуме дискурса $\mathbf{U}$ (фактически расширяя наш универсум до универсума всех неклассических «метамножеств».). Тогда отображение Йонеды будет сопоставлять каждому множеству $\mathbf{S}$ класс-представитель $\hat{S}=\{T \in$ $\left.M: T \in^{*} S\right\}$ относительно нашей «паранепротиворечивой» модели $M$, а наши множества будут в точности теми множествами, которые нужны нам для рассмотрения категории PSet.

ПРЕДЛОЖЕНИЕ 5. PSet является потосом.

Доказательство. Согласно предложению 4 для любого множества $I$ мы всегда имеем (см. [4, р. 84]) паранепротиворечивую алгебру множеств $\left\langle S, \varnothing, I, \leq, \cap, \cup, \Rightarrow{ }^{\prime}\right\rangle$, где $\cap$ и $\cup$ являются теоретико-множественными операциями пересечения и объединения, $\leq$ есть предпорядок, $S \subseteq \wp(I), S$ замкнуто относительно операций $\cap, \cup$ и унарной операции '. Если мы будем рассматривать функции включения как стрелки, то мы можем определить $x \leq y$ тогда и только тогда, когда $x \hookrightarrow y \cup\{b\}$, где $b \in S$. Определим теперь $x^{\prime}=x^{c}$ если $x \notin S_{0}$ и $x^{\prime}=x^{c} \cup \tau$ если $x \in S_{0}$, беря $S_{0}=\{x \in S$ : существует $\tau=\{a, b\}$, такое, что $x \cap \tau \neq \varnothing, x^{c} \cap \tau \neq \varnothing$ и $\left.\neg(x \subset\{a, b\})\right\} \neq \varnothing, x^{c}$ будет теоретикомножественным дополнением $x$. Наконец, определяем $x \Rightarrow y$ как $x^{\prime} \cup y$.

Нетрудно видеть, что в нашей алгебре $x^{o}=I$, если $x \notin S_{0}$, и $x^{o}=I-\{b\}$, если $x \in S_{0}$ для $b \in x \cap \tau$. Наряду с этим $\leq$ является собственным предупорядочением, так как если $b \in x$, то мы имеем $x \cup\{b\} \leq x$ и $x \leq x \cup\{b\}$, но $x \cup\{b\} \neq x$. Следовательно, определяя $x \Leftrightarrow y$ тогда и только тогда, когда $x \leq y$ и $y \leq x$, мы получаем отношение эквивалентности, отличное от равенства. Более того, если $x \subseteq y$ (и таким образом существует стрелка включения $x \hookrightarrow y$ ), то $x \leq y$ и $x=y$ влечет $x \Leftrightarrow y$. Наше отношение эквивалентности $\Leftrightarrow$ несовместимо с ', поскольку, если мы возьмем $x$, такой, что $\neg(\tau \subset x)$, то $x \cup \tau \Leftrightarrow x \cup\{a\}$, где $\tau=\{a, b\}$. Но $(x \cup \tau)^{\prime}=(x \cup \tau)^{c}=x^{c}-\tau$ и $(x \cup\{a\})^{\prime}=(x \cup\{a\})^{c} \cup \tau=x^{c}=\tau$. Таким образом, $x^{c}-\tau \nLeftarrow x^{c} \cup \tau$.

Итак, можно заключить, что в $P S e t$ мы имеем $S u b(d) \cong \wp(d)$ и $S u b(d)$ будет паранепротиворечивой алгеброй множеств, что 
справедливо и для $\wp(d)$. Но в этом случае мы не можем взять 2 в качестве классифицирующего объекта, используя тот факт, что $\wp(d) \cong 2^{d}$, поскольку это приводит к булевой алгебре характеристических стрелок в Set. Действительно, если мы попытаемся определить

$$
\chi_{A}(x)=\left\{\begin{array}{ll}
1, & \text { если } x \in A \\
0, & \text { если } x \notin A
\end{array},\right.
$$

то нам следует принять во внимание, что в PSet у нас имеются два отрицания, и, следовательно, правильное определение будет выглядеть следующим образом:

$$
\chi_{A}(x)= \begin{cases}1, & \text { если } x \in A \\ 2, & \text { если } x \notin A \\ 0, & \text { если } \neg *(x \in A) .\end{cases}
$$

Это означает, что в роли классифицирующего объекта в PSet следует принять не двухэлементную булеву алгебру, но трехэлементную алгебру да Косты (согласно предложению 2 каждая собственная алгебра да Косты имеет по меньшей мере три эламанта). Пример подобной алгебры дается в [4, p. 83], где операции задаются следующими таблицами:

\begin{tabular}{l|lll}
$\wedge$ & 0 & 1 & 2 \\
\hline 0 & 0 & 0 & 0 \\
1 & 0 & 1 & 2 \\
2 & 0 & 2 & 2
\end{tabular}

\begin{tabular}{l|lll}
$\vee$ & 0 & 1 & 2 \\
\hline 0 & 0 & 1 & 2 \\
1 & 1 & 1 & 1 \\
2 & 2 & 1 & 2
\end{tabular}

\begin{tabular}{l|lll}
$\supset$ & 0 & 1 & 2 \\
\hline 0 & 1 & 1 & 1 \\
1 & 0 & 1 & 0 \\
2 & 0 & 1 & 1
\end{tabular}

$0^{\prime}=1,1^{\prime}=0,2^{\prime}=1 ; 0 \leq 2 \leq 1$.

Итак, у нас $\wp(d) \cong 3^{d}$ и множество $3=\{\varnothing,\{\varnothing\},\{\varnothing, 1\}\}$ вместе с функцией true $: 1 \rightarrow 3$ (такой, что $\operatorname{true}(\varnothing)=1$ ) играет роль классификатора подобъектов в PSet. Наряду с этим имеются три стрелки из $1=\{\varnothing\}$ в $\Omega=\{\varnothing, 1,2\}$ : true, false* $: 1 \rightarrow 3$ (такая, что false* $(\varnothing)=\varnothing$ ) и false $: 1 \rightarrow 3$ (такая, что false $(\varnothing)=2$ ).

Q.E.D.

Определим теперь истинностные стрелки в потосе в общем случае. Пусть $\mathbf{C}$ будет потосом с классификатором подобъектов true $: 1 \rightarrow \Omega$. Тогда 
(1) Отрицание $\neg: \Omega \rightarrow \Omega$ будет единственной стрелкой, для которой квадрат

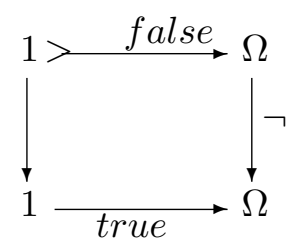

будет декартов в $\mathbf{C}$. Таким образом $\neg=\chi_{\text {false }}$. Отрицание $\neg^{*}$ : $\Omega \rightarrow \Omega$ будет единственной стрелкой, для которой квадрат

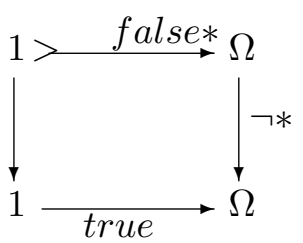

будет декартов в С. В этом случае $\neg^{*}=\chi_{\text {false* }}$

(2) Поскольку потос является декартово замкнутой категорией, то истинностные стрелки определяются стандартным образом:

$\cap: \Omega \times \Omega \rightarrow \Omega$ есть характер произведения стрелок $\langle$ true, true $\rangle$ : $1 \rightarrow \Omega \times \Omega$ в потосе $\mathbf{C}$;

$\cup: \Omega \times \Omega \rightarrow \Omega$ есть по определению характер образа $\mathbf{C}-$ стрелки $\left[\left\langle\right.\right.$ true $\left._{\Omega}, 1_{\Omega}\right\rangle,\left\langle 1_{\Omega}\right.$, true $\left.\left._{\Omega}\right\rangle\right]: \Omega+\Omega \rightarrow \Omega \times \Omega$;

$\Rightarrow: \Omega \times \Omega \rightarrow \Omega$ есть характер монострелки $c: \circledast \mapsto \Omega \times \Omega$, которая является уравнителем пары

$$
\Omega \times \Omega \underset{p r_{1}}{\stackrel{\cap}{\rightrightarrows}} \Omega
$$

где $p r_{1}$ есть проекция на первый сомножитель произведения $\Omega \times$ $\Omega$. 


\section{4 Интерпретация паранепротиворечивой логики в потосах}

Дадим интерпретацию в терминах потоса следующего списка аксиом и правил вывода [5, p. 3790]:

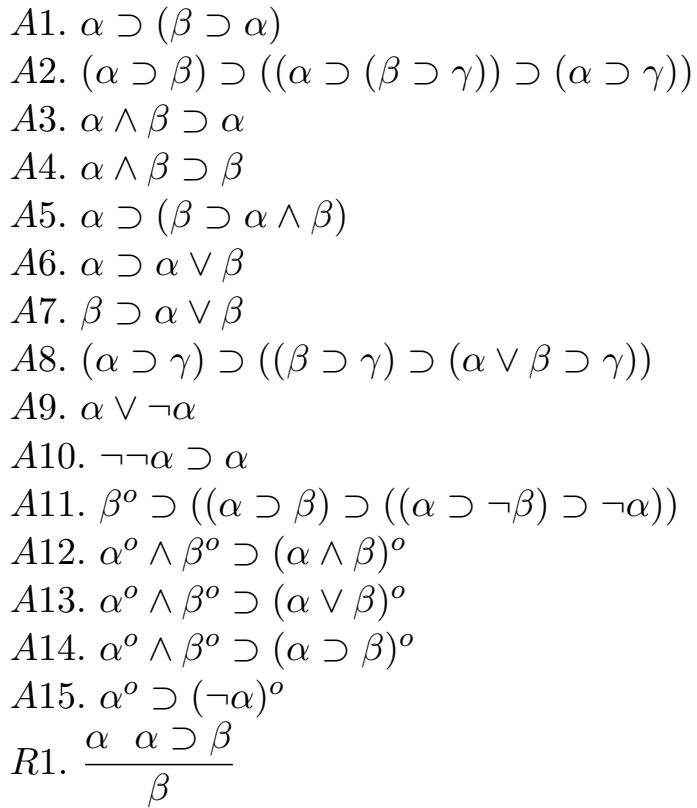

Здесь $\alpha^{o}$ является сокращением для $\neg(\alpha \vee \neg \alpha)$. Данная аксиоматика фактически описывает систему $C_{1}$ паранепротиворечивой логики да Косты.

Мы получим исчисление $C_{n}(1 \leq n \leq \omega)$, если вместо аксиом $A 11-A 15$ используем следующие аксиомы:

$A 11^{\prime} . \beta^{(n)} \supset((\alpha \supset \beta) \supset((\alpha \supset \neg \beta) \supset \neg \alpha))$

$A 12^{\prime} . \alpha^{(n)} \wedge \beta^{(n)} \supset(\alpha \wedge \beta)^{(n)}$

$A 13^{\prime} . \alpha^{(n)} \wedge \beta^{(n)} \supset(\alpha \vee \beta)^{(n)}$

$A 14^{\prime} . \alpha^{(n)} \wedge \beta^{(n)} \supset(\alpha \supset \beta)^{(n)}$

$A 15^{\prime} . \alpha^{(n)} \supset(\neg \alpha)^{(n)}$

где $\alpha^{(n)}(1 \leq n \leq \omega)$ означает $\alpha^{1} \wedge \alpha^{2} \wedge \ldots \wedge \alpha^{n}$, а $\alpha^{n}$, в свою очередь, означает $\alpha^{\text {оо..o }}$, где символ ${ }^{o}$ повторяется $n$ раз.

Можно определить оценку $v: \Phi_{0} \rightarrow A$ системы $C_{1}$ в алгебре да Косты $A$, ставящую в соответствие каждой пропозициональной переменной $\pi_{i}$ некоторое истинностное значение $V\left(\pi_{i}\right) \in A$. Она однозначно продолжается по следующим правилам:

(1) $v(\neg \alpha)=v(\alpha)^{\prime}$; 
(2) $v(\alpha \wedge \beta)=v(\alpha) \wedge v(\beta)$;

(3) $V(\alpha \vee \beta)=v(\alpha) \vee v(\beta)$;

(4) $V(\alpha \supset \beta)=v(\alpha) \supset v(\beta)$,

до функции $v: \Phi \rightarrow A$. Предложение $\alpha$, такое, что $v(\alpha)=1$ для каждой $A$-оценки $v$ называется $A$-общезначимым и обозначают это через $A \models \alpha$.

TEOРЕМА 3. Для любой алгебры да Косты $A$ имеет место $\vdash_{C_{1}} A$ тогда и толъко тогда, когда $A \models \alpha$.

Доказательство. Слева направо проверяется $C_{1}$-общезначимость всех $C_{1}$-аксиом и правила отделимости. Чтобы получить доказательство утверждения справа налево, воспользуемся теоремой 2. Сопоставим каждому элементу $x$ алгебры $A$ главный фильтр $[x)=\{q: x \leq q\}$, где $q \in A$ Алгебра $A^{+}$главных фильтров будет алгеброй да Косты. Определим $A^{+}$-оценку как функцию $v_{c}: \Phi_{0} \rightarrow A^{+}$с помощью формулы $v_{\mathrm{c}}\left(\pi_{i}\right)=\left[v\left(\pi_{i}\right)\right)$. Остальное очевидно.

Q.E.D.

Определим теперь интерпретацию рассматриваемой системы в произвольном потосе $C$. Истинностным значением в потосе называется стрелка вида $1 \rightarrow \Omega$, а совокупность всех таких $C$ стрелок будет представлять собой множество стрелок $C(1, \Omega)$.

$C$-оценкой будем называть функцию $V: \Phi_{0} \rightarrow C(1, \Omega)$, ставящую в соответствие каждой пропозицииональной переменной $\pi_{i}$ некоторуое истинностное значение $V\left(\pi_{i}\right): 1 \rightarrow \Omega$. Эта функция продолжается на множество всех формул $\Phi$ по следующим правилам:

(a) $V(\neg \alpha)=\neg \circ V(\alpha)$;

(b) $V(\alpha \wedge \beta)=\cap \circ\langle V(\alpha), V(\beta)\rangle$;

(c) $V(\alpha \vee \beta)=\cup \circ\langle V(\alpha), V(\beta)\rangle$;

(d) $V(\alpha \supset \beta)=\Rightarrow \circ\langle V(\alpha), V(\beta)\rangle$.

Таким образом мы продолжаем оценку $V$ так, что каждому предложению $\alpha$ ставится в соответствие некоторая $C$-стрелка $V(\alpha): 1 \rightarrow \Omega$. $C$-общезначимость предложения $\alpha$ (что обозначается как $C=\alpha$ ) означает, что $V(\alpha)=$ true $: 1 \rightarrow \Omega$ для всех оценок $V$.

Поскольку в потосе мы имеем $\operatorname{Sub}(d) \cong \operatorname{Hom}(d, \Omega)$, то отсюда $S u b(d) \cong C(d, \Omega)$, т.е. ставя подобъекту $f$ его характер $\chi_{f}$, 
мы получаем перенос структуры алгебры да Косты с $S u b(d)$ на $C(d, \Omega)$. Связь между семантикой потосов и рассматриваемой теорией, как и в случае алгебры Гейтинга [2, с. 199], заключается в том, что для любого потоса

$C \models \alpha$ тогда и только тогда, когда $C(1, \Omega) \models \alpha$ тогда и только тогда, когда $S u b(1) \models \alpha$.

Таким образом, общезначимость в потосе $C$ равносильна общезначимости в алгебрах да Косты $C(1, \Omega)$ и $S u b(1)$. Отсюда получаем следующую теорему:

TEOРЕМА 4. Если $\vdash_{C_{1}} \alpha$, то в любом потосе $\mathrm{C}$ имеем $\mathrm{C} \models \alpha$.

Доказательство. Пусть $\alpha$ - некоторая $C_{1}$-теорема. Тогда $\alpha$ общезначима в алгебре да Косты по теореме 3 . В частности, $C(1, \Omega) \models \alpha$, откуда $C \models \alpha$ согласно предыдущему утверждению.

Существует еще один способ доказательства предыдущей теоремы, если воспользоваться неистинностно-функциональной оценкой для системы $C_{1}$. Согласно [6] мы можем ввести оценку $V^{\prime}: \Phi_{0} \rightarrow\{0,1\}$, где $\Phi_{0}$ есть множество пропозициональных переменных, и расширить ее на множество $\Phi$ всех формул следующим образом:

(1) $V^{\prime}(\alpha)=0 \Rightarrow V^{\prime}(\neg \alpha)=1$

(2) $V^{\prime}(\neg \neg \alpha)=1 \Rightarrow V^{\prime}(\alpha)=1$;

(3) $V^{\prime}\left(\alpha^{\circ}\right)=V^{\prime}(\alpha \supset \beta)=V^{\prime}(\alpha \supset \neg \beta)=1 \Rightarrow V^{\prime}(\alpha)=0$;

(4) $V^{\prime}(\alpha \supset \beta)=1 \Leftrightarrow V^{\prime}(\alpha)=0$ or $V^{\prime}(\beta)=1$;

(5) $V^{\prime}(\alpha \wedge \beta)=1 \Leftrightarrow V^{\prime}(\alpha)=V^{\prime}(\beta)=1$;

(6) $V^{\prime}(\alpha \vee \beta)=1 \Leftrightarrow V^{\prime}(\alpha)=1$ or $V^{\prime}(\beta)=1$;

(7) $V^{\prime}\left(\alpha^{\circ}\right)=V^{\prime}\left(\beta^{\circ}\right)=1 \Rightarrow V^{\prime}\left((\alpha \supset \beta)^{\circ}\right)=V^{\prime}\left((\alpha \wedge \beta)^{\circ}\right)=$ $V^{\prime}\left((\alpha \vee \beta)^{\circ}\right)=1$.

Мы можем сопоставить $V$ оценку $V^{\prime}$, полагая $V\left(\pi_{i}\right)=t r u e$, если $V^{\prime}\left(\pi_{i}\right)=1$, и $V\left(\pi_{i}\right)=$ false в противном случае.

ЛЕММА 1. $V(\alpha)=$ trие тогда и только тогда, когда $V^{\prime}(\alpha)=1$.

Доказательство. В случае $\alpha=\pi_{i}$ лемма справедлива по определению. Используем индукцию по длине формулы, т.е. используем (1)-(7). Пусть $\alpha=\neg \beta$ и $V(\beta)=$ false. Тогда

$V(\neg \beta)=\neg \circ V(\beta)$. 
Следовательно, $V(\alpha)(0)=\neg(V(\beta)(0))=\neg($ false $(0))=($ по индуктивному определению) $=\operatorname{true}(0)$. Таким образом, $V(\alpha)=$ true и $V^{\prime}(\alpha)=1$. Остальное получаем действуя аналогичным образом.

Q.E.D.

Q.E.D.

TEОРЕМА 5. Для любого потоса $\mathrm{C}$ и пропозициональной формулы $\alpha$ имеет место $\mathrm{C} \models \alpha$ тогда и толъко тогда, когда $\vdash_{C_{1}} \alpha$.

Доказательство. Поскольку по лемме 1 мы выбираем оценку $V$ абсолютно произвольным образом, а для $V^{\prime}$ в [6] полнота уже доказана, то это и приводит к требуемому заключению. Q.E.D.

\section{Литература}

[1] Васюков В.Л. Потосы для паранепротиворечивой логики // Современная логика: проблемы теории, истории и применения в науке. Материалы Х Общероссийской научн. конференции, СПб., 2008. С.105-107.

[2] Голдблатm P. Топосы. Категорный анализ логики. М., 1983.

[3] J.Benabou. Fibered Categories and the Foundations of Naive Category Theory // The Journal of Symbolic Logic,50(4):9-37, 1983.

[4] W.A. Carnielli, L.P. Alcantara. Paraconsistent algebras // Studia Logica, XLIII, No $1 / 2$, 1984, pp. 79-87.

[5] N.C.A.da Costa. Calculus propositionnels pour les systemes inconsistants // C.R.Acad.Sci.Paris, T.257, 3790-3792, 1963.

[6] N.C.A.da Costa and E.H.Alves. Une semantique pour le calculi $C_{1} / /$ C.R.Acad.Sci.Paris, T.283, 729-731, 1976.

[7] N.C.A.da Costa. Paraconsistent Mathematics // D.Batens, C.Mortensen, G.Priest and J.-P. van Bendegem (eds.), Frontiers of Paraconsistent Logic, Research Studies Press Ltd., Baldock, Hartfordshire, England, 2000, pp. 166-179.

[8] V.L. Vasyukov. Paraconsistency in Categories // D.Batens, C.Mortensen, G.Priest and J.-P. van Bendegem (eds.), Frontiers of Paraconsistent Logic, Research Studies Press Ltd., Baldock, Hartfordshire, England, 2000, pp. 263-278. 